\title{
CURE-Chloroplast: A chloroplast C-to-U RNA editing predictor for seed plants
}

Pufeng Duं, Liyan Jia ${ }^{\dagger}$ and Yanda $\mathrm{Li}^{*}$

\author{
Address: MOE Key Laboratory of Bioinformatics and Bioinformatics Div. TNLIST/Department of Automation, Tsinghua University, Beijing \\ 100084, PR China \\ Email: Pufeng Du -dpf05@mails.tsinghua.edu.cn; Liyan Jia - jly05@mails.tsinghua.edu.cn; Yanda Li* - daulyd@tsinghua.edu.cn \\ * Corresponding author †Equal contributors
}

Published: 8 May 2009

BMC Bioinformatics 2009, 10:135 doi:10.1/86/1471-2105-10-135

This article is available from: http://www.biomedcentral.com/I47/-2/05/I0//35

(c) 2009 Du et al; licensee BioMed Central Ltd.

This is an Open Access article distributed under the terms of the Creative Commons Attribution License (http://creativecommons.org/licenses/by/2.0), which permits unrestricted use, distribution, and reproduction in any medium, provided the original work is properly cited.
Received: 23 October 2008

Accepted: 8 May 2009

\begin{abstract}
Background: RNA editing is a type of post-transcriptional modification of RNA and belongs to the class of mechanisms that contribute to the complexity of transcriptomes. C-to-U RNA editing is commonly observed in plant mitochondria and chloroplasts. The in vivo mechanism of recognizing C-to-U RNA editing sites is still unknown. In recent years, many efforts have been made to computationally predict C-to-U RNA editing sites in the mitochondria of seed plants, but there is still no algorithm available for C-to-U RNA editing site prediction in the chloroplasts of seed plants.

Results: In this paper, we extend our algorithm CURE, which can accurately predict the C-to-U RNA editing sites in mitochondria, to predict C-to-U RNA editing sites in the chloroplasts of seed plants. The algorithm achieves over $80 \%$ sensitivity and over $99 \%$ specificity. We implement the algorithm as an online service called CURE-Chloroplast http://bioinfo.au.tsinghua.edu.cn/pure.

Conclusion: CURE-Chloroplast is an online service for predicting the C-to-U RNA editing sites in the chloroplasts of seed plants. The online service allows the processing of entire chloroplast genome sequences. Since CURE-Chloroplast performs very well, it could be a helpful tool in the study of C-to-U RNA editing in the chloroplasts of seed plants.
\end{abstract}

\section{Background}

RNA editing is a kind of RNA processing (like splicing, 5 'capping and 3 ' polyadenylation) that can alter the genetic information of RNA via insertion, deletion or substitution of single or multiple nucleotides. In plant mitochondrial and chloroplast transcripts, several cytidines can be converted to uridines by a deamination process [13]. Generally, there are about 300 to 500 C-to-U RNA editing sites in the mitochondrial transcriptomes of seed plants [4-7], but only 30 to 50 can be found in their chloroplasts [8-12]. Most of the known C-to-U RNA editing instances in plant organelles share a similar property: they are non-synonymous and alter the encoded protein sequence to be more conserved across species than the protein sequence predicted from genomic DNA [13-16]. This effect makes the actual protein sequence different from the sequence predicted from the genomic DNA. Thus, knowledge of C-to-U RNA editing in plant organelles is important in order to correctly analyze the protein sequence and gene expression in both computational and experimental studies.

Biologically, the in vivo site recognition mechanism of Cto-U RNA editing in plant organelles is still not fully understood [17]. While several short sequences in the upstream regions of the editing sites have been identified 
as being critical for site recognition [18-22], little is known about the factors that recognize these sites. Recent studies have suggested that the PPR family of proteins is related to site recognition [23-26]. It seems that every single site or every small set of editing sites are recognized by a specific factor $[27,28]$. With the above biological knowledge as a basis, machine learning algorithms were introduced to predict C-to-U RNA editing sites in mitochondria. Cummings and Myers proposed the first prediction algorithm for C-to-U RNA editing sites in mitochondria based on the classification tree algorithm [29], REGAL introduced the genetic algorithm $[30,31]$ and Prep-Mt [32] and our CURE (Cytidine-to-Uridine Recognizing Editor) algorithm [33] considered the evolutionary information. Yura et al. proposed a method (RNAE) for predicting the C-to-U RNA editing sites in the chloroplasts of one particular moss organism, Takakia lepidozioides [34]. However, the application of RNAE on the seed plant chloroplast genes results in very poor accuracy (Additional file 1). In other words, RNAE is designed for only one moss organism but cannot be used as a predictor for seed plant organisms.

Although the chloroplast C-to-U RNA editing sites of rice [35], maize [8], tobacco [10], sugarcane [36], peas [37], orchids [38] and several other seed plants have been systematically determined through experiments, there is still no available software for predicting the chloroplast C-toU RNA editing sites in seed plants. This may be due to the relatively small number of editing instances in the chloroplast transcriptomes of these organisms, which makes algorithm design and evaluation very difficult.

However, in the public sequence database, there are many chloroplast transcripts with undetermined RNA editing status. An accurate computational prediction method would be helpful in filling the gap between the sequence data and the RNA editing annotations. It would also reduce the need for experimental determinations, which are costly and time consuming. In this paper, we will extend our algorithm CURE, which can accurately predict C-to-U RNA editing sites in mitochondria, to predict C-toU RNA editing sites in the chloroplasts of seed plants. We will also present the online prediction service, CUREChloroplast.

\section{Implementation \\ Dataset}

There are three main RNA editing databases: dbRES [39], REDIdb [40] and EdRNA [41]. REDIdb is the only database focusing on organelle RNA editing sites. We collected all the C-to-U RNA editing sites of seed plant chloroplasts in REDIdb. The duplicate annotations in the database were discarded. The inaccurate annotations, which were associated with nucleotides other than cytidine, were cor- rected according to the original literatures or the GenBank annotations. The editing sites in pea [37], sugarcane [36] and orchid [38] chloroplasts were extracted from the literatures and added into the dataset. The sequences in this dataset were categorized by the gene name. ClustalW was used to create alignments for each gene. These alignments were used to train CURE-Chloroplast. Table 1 shows the summary of our dataset.

This dataset has significant lineage bias. Most of the editing sites are from angiosperms. Only one gymnosperm plant is included. Since several editing sites in the chloroplasts of the gymnosperm are not conserved in angiosperms [9], we need to develop different strategies to predict the C-to-U RNA editing sites in angiosperms and gymnosperms.

\section{The basic CURE-Chloroplast algorithm}

The basic algorithm for CURE-Chloroplast is the same as the CURE algorithm, which we have already successfully developed to predict mitochondria C-to-U RNA editing sites [33]. The CURE algorithm was based on the fact that if one editing site can be found in a column of a multiple sequence alignment of homologous genes from different organisms, it is likely to find another in the same column. In the CURE algorithm, we proposed the concept of Evolutionary Potential Editing Sites (EPESs). In a multiple sequence alignment, if a column contains an editing site, this column is defined as an EPES. An EPES is described with three different elements: a flanking consensus sequence, a conservative ratio and a set of sequences that generate the flanking consensus sequence. The training procedure scans the alignments to collect the EPESs and save them in a database. When the editing sites of a sequence need to be predicted, the prediction procedure

Table I: The summary of the dataset

\begin{tabular}{ccccc}
\hline Organism & No. of Genes & Total & POS & NEG \\
\hline Arabidopsis thaliana & 13 & 2284 & 28 & 2256 \\
Atropa belladonna & 14 & 2885 & 27 & 2858 \\
Nicotiana sylvestris & 17 & 1960 & 35 & 1925 \\
Nicotiana tabacum & 17 & 3712 & 32 & 3680 \\
Nicotiana tomentosiformis & 16 & 1921 & 33 & 1888 \\
Oryza sativa & 10 & 2362 & 20 & 2342 \\
Phalaenopsis aphrodite & 22 & 3802 & 42 & 3760 \\
Pinus thunbergii & 13 & 1658 & 28 & 1630 \\
Pisum sativum & 16 & 2839 & 26 & 2813 \\
Saccharum officinarum & 13 & 3311 & 23 & 3288 \\
Zea mays & 13 & 3294 & 25 & 3269 \\
Overall & 164 & 30028 & 319 & 29709 \\
\hline
\end{tabular}

The Number of Genes column is the number of edited genes in the organism, the Total column is the number of all cytidines in the edited genes, the POS column is the number of edited cytidines in the edited genes and the NEG column is the number of unedited cytidines in the edited genes. 
uses the BLAST program to map the EPES consensus sequences in the database to this sequence and determines the editing status of every cytidine. The details of the basic CURE algorithm can be found either in our previous work [33] or in additional file 2 of the current paper.

The alignments for training can be prepared by two different methods. One is to create alignments from the CDS sequences of each gene with the ClustalW program; the other is to create alignments directly from the whole genome sequences using the TBA program [42]. Since most of the RNA editing sites in chloroplasts are found within the coding regions, we only use the former method to prepare the alignments. However, CURE-Chloroplast users are still allowed to enter nucleotide sequences without any restrictions - even the entire chloroplast genome sequence can be directly entered. In this situation, only those RNA editing sites in gene regions can be identified because our training dataset contains no information from the non-coding regions.

\section{CURE-Chloroplast algorithm extended for gymnosperms}

As we have mentioned in the dataset section, several editing sites in gymnosperms are not conserved to the angiosperms. Thus, we need to modify the CURE algorithm to make it applicable to gymnosperms. An extended definition of an EPES was proposed. If a column in the multiple sequence alignments contains only $\mathrm{T}$, and this column is at the middle position of a codon, this column is also defined as an EPES. This extended EPES definition is based on the fact that the protein translated from the edited version of the transcript is usually more conserved across species than the sequence predicted from the genomic DNA. Thus, if a cytidine is mapped by this extended EPES, it is likely to be edited to restore the conservation at the protein level.

\section{Evaluation}

Six summary statistics were used to describe the performance of CURE-Chloroplast: sensitivity, specificity, accuracy, positive predictive value (PPV), balanced accuracy (BA) and the Matthew's correlation coefficient (MCC) (Eq. 1 Eq. 6).

$$
\begin{gathered}
\text { Sensitivity }=\frac{T P}{T P+F N} \\
\text { Specificity }=\frac{T N}{T N+F P} \\
\text { Accuracy }=\frac{T P+T N}{T P+T N+F P+F N} \\
P P V=\frac{T P}{T P+F P}
\end{gathered}
$$

$$
\begin{gathered}
B A=\frac{1}{2}(\text { Specificity }+ \text { Sensitivity }) \\
M C C=\frac{T P \cdot T N-F P \cdot F N}{\sqrt{(T P+F P) \cdot(T P+F N) \cdot(T N+F N) \cdot(T N+F P)}}
\end{gathered}
$$

$\mathrm{TP}, \mathrm{TN}, \mathrm{FP}$ and $\mathrm{FN}$ are the number of true positives, true negatives, false positives and false negatives, respectively.

\section{Online Service}

CURE-Chloroplast was implemented on a Linux server with two Xeon dual-core CPUs and 4 GB of memory. Users can either paste a single sequence or upload a FASTA file containing multiple sequences via the web-based user interface. The server-side PHP scripts process the sequences and carry out the prediction. The results are presented in a web-based result browser. The plain text-based result file can be downloaded within 24 hours after the prediction is made.

CURE-Chloroplast users are allowed to adjust several parameters after the advanced mode is enabled. The "Up Bound" and "Low Bound" parameters define the working region of a K-NN classifier, which is called a "micro-analyzer" in the CURE-Chloroplast system. When the submitted sequence is mapped by an EPES with an RNA editing conservative ratio between the Low Bound and Up Bound, the K-NN classifier will be used to decide whether or not the EPES mapping cytidine should be predicted as an editing site. If the users enlarge the working region bounded by these two parameters, the editing statuses of more cytidines are determined by the K-NN classifier rather than the RNA editing conservative ratio. According to our experience, the default parameters are suitable for most organisms.

The CURE-Chloroplast service has two more options than the CURE service. One is the "Cons-T EPES" option. If this option is turned on, the extended EPES definition will be used. The other is the "Positive strand only" option. If this option is turned on, CURE-Chloroplast will only scan the positive strand of the input sequence.

\section{Results and Discussion \\ Prediction performance analysis}

We used leave-one-species-out cross-validation to estimate the performance of CURE-Chloroplast. When we were testing the performance on one organism, all the data relating to that organism, including sequences and editing sites, were removed from the training set. The algorithm was retrained on the remaining data. The details of CURE-Chloroplast performance can be found in Table 2. Overall, CURE-Chloroplast achieved over $80 \%$ 
sensitivity and over 99\% specificity. Although the negatives were much more than the positives in the dataset, we found that the sensitivity was still acceptable for most species. CURE-Chloroplast can identify the tiny number of positives among the extremely large number of negatives. Because the dataset was significantly unbalanced, we provided the PPV and MCC values as measures of performance on the unbalanced dataset. Since a similar performance estimation problem in Prep-Mt was solved by introducing balanced accuracy statistics [32], we also provided the balanced accuracy values. The balanced accuracy can be considered as the estimation of accuracy on a balanced dataset.

The extended EPES definition was applied for gymnosperm plants. With the extended EPES definition, the sensitivity on Pinus thunbergii was more than twice that of the original definition, the PPV only decreased by about $20 \%$. The extended EPES definition successfully improved the performance on gymnosperms. All the following tests on gymnosperms were carried out using the extended EPES definition.

The pioneer research on predicting the C-to-U RNA editing sites in mitochondria was carried out on the balanced dataset [29]. We also built a similar balanced dataset to test the performance of our algorithm. We randomly selected a set of negatives with an equal number of positives. This set of negatives and all the positives composed the balanced dataset. The performance on this balanced dataset was almost unchanged (Table 3 ). The accuracy of this balanced dataset was found to be similar to the balanced accuracy of an unbalanced dataset, indicating the accuracy of a balanced dataset can be estimated by the balanced accuracy of an unbalanced dataset.
To further eliminate the concerns of over-fitting algorithm, we carried out an independent data test. We randomly selected $10 \%, 20 \%$ and $30 \%$ of the dataset as the test samples. The remaining data were used as the training set. The performance estimated with this independent test was similar to the leave-one-species-out cross-validation performance (Table 4), indicating the performance of the algorithm was not over-estimated.

It should be noted that the RNA editing sites of the chloroplast genes of two parasitic flowering plants, Cuscuta reflexa and Cuscuta gronovii, were recently determined [43]. These data were not deposited in the public databases and were not considered when we were developing CURE-Chloroplast. CURE-Chloroplast identified all 15 known editing sites with only two false positives in Cuscuta reflexa and three of the four known editing sites with only three false positives in Cuscuta gronovii. The overall performance in this full-blind validation achieved 94.7\% sensitivity, $99.8 \%$ specificity, $78.3 \%$ PPV and $99.7 \%$ accuracy.

The parameters of CURE-Chloroplast can be adjusted in the same manner as for CURE [33]. We set the default parameters of CURE-Chloroplast to the same values as CURE. These parameters worked well enough throughout our tests. In addition, we calculated the performance of CURE-Chloroplast on Arabidopsis thaliana with different parameters and illustrated an ROC-like curve (Additional file 3) that describes the performance under different parameter conditions.

\section{Performance on entire chloroplast genome sequences}

As we have described in the Implementation section, CURE-Chloroplast can directly process the entire genome

Table 2: The performance of leave-one-species-out cross-validation

\begin{tabular}{|c|c|c|c|c|c|c|}
\hline Organism & Sen & Spe & PPV & $\mathrm{ACC}$ & $\mathrm{BA}$ & $\mathrm{MCC}$ \\
\hline Arabidopsis thaliana & $71.43 \%$ & $99.87 \%$ & $86.96 \%$ & $99.52 \%$ & $85.65 \%$ & 0.79 \\
\hline Atropa belladonna & $92.59 \%$ & $99.79 \%$ & $80.65 \%$ & $99.72 \%$ & $96.19 \%$ & 0.86 \\
\hline Nicotiana sylvestris & $91.43 \%$ & $99.90 \%$ & $94.12 \%$ & $99.74 \%$ & $95.66 \%$ & 0.93 \\
\hline Nicotiana tabacum & $90.63 \%$ & $99.84 \%$ & $82.86 \%$ & $99.76 \%$ & $95.23 \%$ & 0.87 \\
\hline Nicotiana tomentosiformis & $90.91 \%$ & $99.74 \%$ & $85.71 \%$ & $99.58 \%$ & $95.32 \%$ & 0.88 \\
\hline Oryza sativa & $100.00 \%$ & $99.87 \%$ & $86.96 \%$ & $99.87 \%$ & $99.94 \%$ & 0.93 \\
\hline Phalaenopsis aphrodite & $40.48 \%$ & $99.89 \%$ & $80.95 \%$ & $99.24 \%$ & $70.18 \%$ & 0.57 \\
\hline Pinus thunbergii $(*)$ & $64.29 \%$ & $99.02 \%$ & $52.94 \%$ & $98.43 \%$ & $81.65 \%$ & 0.58 \\
\hline Pinus thunbergii & $28.57 \%$ & $99.82 \%$ & $72.73 \%$ & $98.61 \%$ & $64.19 \%$ & 0.45 \\
\hline Pisum sativum & $76.92 \%$ & $99.75 \%$ & $74.07 \%$ & $99.54 \%$ & $88.34 \%$ & 0.75 \\
\hline Saccharum officinarum & $100.00 \%$ & $99.91 \%$ & $88.46 \%$ & $99.91 \%$ & $99.95 \%$ & 0.94 \\
\hline Zea mays & $96.00 \%$ & $99.97 \%$ & $96.00 \%$ & $99.94 \%$ & $97.98 \%$ & 0.96 \\
\hline Over All & $80.88 \%$ & $99.81 \%$ & $82.17 \%$ & $99.61 \%$ & $90.34 \%$ & 0.81 \\
\hline
\end{tabular}

Sen means sensitivity, Spe means specificity, PPV means positive predictive value, ACC means accuracy, BA means balanced accuracy and MCC means Matthew's correlation coefficient. All the values were obtained with leave-one-species-out cross-validation on the training set. The performance marked with "(*)" was obtained using the extended EPES definition. The overall performance was calculated using the "(*)" performance. 
Table 3: The performance evaluated on a balanced dataset

\begin{tabular}{|c|c|c|c|c|c|c|}
\hline Organism & Sen & Spe & PPV & $\mathrm{ACC}$ & $\mathrm{BA}$ & $\mathrm{MCC}$ \\
\hline Arabidopsis thaliana & $71.43 \%$ & $100.00 \%$ & $100.00 \%$ & $85.71 \%$ & $85.71 \%$ & 0.75 \\
\hline Atropa belladonna & $92.59 \%$ & $100.00 \%$ & $100.00 \%$ & $96.30 \%$ & $96.30 \%$ & 0.93 \\
\hline Nicotiana sylvestris & $91.43 \%$ & $100.00 \%$ & $100.00 \%$ & $95.71 \%$ & $95.71 \%$ & 0.92 \\
\hline Nicotiana tabacum & $90.63 \%$ & $100.00 \%$ & $100.00 \%$ & $95.31 \%$ & $95.31 \%$ & 0.91 \\
\hline Nicotiana tomentosiformis & $90.91 \%$ & $100.00 \%$ & $100.00 \%$ & $95.45 \%$ & $95.45 \%$ & 0.91 \\
\hline Oryza sativa & $100.00 \%$ & $100.00 \%$ & $100.00 \%$ & $100.00 \%$ & $100.00 \%$ & 1.00 \\
\hline Phalaenopsis aphrodite & $40.48 \%$ & $100.00 \%$ & $100.00 \%$ & $70.24 \%$ & $70.24 \%$ & 0.50 \\
\hline Pinus thunbergii & $64.29 \%$ & $100.00 \%$ & $100.00 \%$ & $82.14 \%$ & $82.14 \%$ & 0.69 \\
\hline Pisum sativum & $76.92 \%$ & $100.00 \%$ & $100.00 \%$ & $88.46 \%$ & $88.46 \%$ & 0.79 \\
\hline Saccharum officinarum & $100.00 \%$ & $100.00 \%$ & $100.00 \%$ & $100.00 \%$ & $100.00 \%$ & 1.00 \\
\hline Zea mays & $96.00 \%$ & $100.00 \%$ & $100.00 \%$ & $98.00 \%$ & $98.00 \%$ & 0.96 \\
\hline Over All & $80.88 \%$ & $100.00 \%$ & $100.00 \%$ & $90.44 \%$ & $90.44 \%$ & 0.82 \\
\hline
\end{tabular}

Sen means sensitivity, Spe means specificity, PPV means positive predictive value, ACC means accuracy, BA means balanced accuracy and MCC means Matthew's correlation coefficient. On the balanced dataset, the BA always equals the ACC.

sequence. Four organisms, including Arabidopsis thaliana, Nicotiana tabacum, Zea mays and Pinus thunbergii, were chosen to test the performance of CURE-Chloroplast under this condition. These organisms were chosen because they are representative of their lineages. When an organism was used as the testing sample, all the information relating to this organism was removed from the training set. The entire chloroplast genome sequence of that organism was used as the testing sequence. The results are shown in Table 5 . The overall sensitivity is still over $70 \%$ under this condition. The PPV decreased because it is difficult to prevent the increment of the number of false positives when the number of negatives for testing is over 100,000 and the number of positives for testing is only about 100 . The performance under this condition can be considered as a good estimation of the performance that a real user may experience, as this test condition simulates the practical application of CURE-Chloroplast.

\section{Cross-prediction between CURE and CURE-Chloroplast}

The in vivo site recognition mechanism of the C-to-U RNA editing sites is still not understood. One hypothesis is that every single editing site or small set of editing sites is recognized by a particular factor $[22,28]$. The sequence patterns of the chloroplast C-to-U RNA editing sites are not expected to be shared with the mitochondria editing sites.

Table 4: The performance in independent tests

\begin{tabular}{ccccccc}
\hline Test data & Sen & Spe & PPV & ACC & BA & MCC \\
\hline $10 \%$ & $86.67 \%$ & $99.74 \%$ & $68.42 \%$ & $99.66 \%$ & $93.20 \%$ & 0.77 \\
$20 \%$ & $88.57 \%$ & $99.68 \%$ & $72.09 \%$ & $99.58 \%$ & $94.13 \%$ & 0.80 \\
$30 \%$ & $79.83 \%$ & $99.75 \%$ & $77.50 \%$ & $99.54 \%$ & $89.79 \%$ & 0.78 \\
\hline
\end{tabular}

Sen means sensitivity, Spe means specificity, PPV means positive predictive value, ACC means accuracy, BA means balanced accuracy and MCC means Matthew's correlation coefficient. The Test data column is the percentage of data that has been randomly selected as the test set. The remaining data are used as the training set.
Thus, the model trained from mitochondria and chloroplasts should not work for each other. Since we have developed CURE for predicting mitochondrial editing sites and CURE-Chloroplast for predicting chloroplast editing sites, we can see what will happen if we use the model trained on mitochondria and chloroplasts to predict the editing sites of each other.

We took Arabidopsis thaliana as an example. We use CURE to predict the editing sites on all edited genes in the chloroplasts and CURE-Chloroplast to predict the editing sites on all edited genes in the mitochondria. Both tests returned nothing. The model trained on chloroplasts and mitochondria cannot be used to predict the editing sites of each other. These results agree with the one-site-one-factor hypothesis.

\section{Can CURE-Chloroplast work on non-seed plants?}

C-to-U RNA editing prediction in non-seed plants is a special problem for chloroplasts. All prediction algorithms for plant mitochondria were developed and tested with the editing sites of seed plants. Although C-to-U RNA editing sites exist in the mitochondria of non-seed plants, no computational prediction algorithm takes them into consideration.

The situation of chloroplast C-to-U RNA editing is a bit different. There are over 300 editing sites in the fern Adiantum capillus-veneris [44] and over 500 editing sites in the hornwort Anthoceros formosae [45]. The editing patterns of these two organisms are significantly different from each other and significantly different from those of seed plants. Most of the C-to-U RNA editing sites in these two species are not conserved in seed plants [44]. With the extended EPES definition, CURE-Chloroplast can achieve sensitivity $39 \%$ for Adiantum capillus-veneris and $51 \%$ for Anthoceros formosae, specificity $88 \%$ for Adiantum capillusveneris and $86 \%$ for Anthoceros formosae, PPV 6\% for Adi- 
Table 5: Performance test with the entire genome sequence

\begin{tabular}{|c|c|c|c|c|c|c|}
\hline Organism & Sen & Spe & PPV & $\mathrm{ACC}$ & BA & $\mathrm{MCC}$ \\
\hline Arabidopsis thaliana & $67.86 \%$ & $99.93 \%$ & $48.72 \%$ & $99.90 \%$ & $83.89 \%$ & 0.57 \\
\hline Nicotiana tabacum & $87.50 \%$ & $99.95 \%$ & $65.12 \%$ & $99.94 \%$ & $93.72 \%$ & 0.75 \\
\hline Pinus thunbergii & $50.00 \%$ & $99.23 \%$ & $7.29 \%$ & $99.17 \%$ & $74.61 \%$ & 0.19 \\
\hline Zea Mays & $84.00 \%$ & $99.94 \%$ & $58.33 \%$ & $99.93 \%$ & $91.97 \%$ & 0.70 \\
\hline Over all & $72.57 \%$ & $99.79 \%$ & $26.45 \%$ & $99.76 \%$ & $86.18 \%$ & 0.44 \\
\hline
\end{tabular}

Sen means sensitivity, Spe means specificity, PPV means positive predictive value, ACC means accuracy, BA means balanced accuracy and MCC means Matthew's correlation coefficient.

antum capillus-veneris and 14\% for Anthoceros formosae and accuracy $87 \%$ for Adiantum capillus-veneris and $85 \%$ for Anthoceros formosae. The performance is acceptable, but lower than the performance in seed plants.

It has been suggested that the editing sites in these organisms and the seed plants are of monophyletic origin [46]. Thus, the CURE-Chloroplast algorithm should work well on these organisms. However, there are other reasons preventing CURE-Chloroplast from working well on these organisms. Technically, CURE-Chloroplast relies on accurate sequence alignment while mapping the EPESs on the target sequence. The sequence divergence between the homologous genes of these organisms and the seed plants makes it very difficult to map the EPES trained from the seed plants to the sequence of these two far related organisms.

Another more telling explanation for the low performance of CURE-Chloroplast with regard to these two organisms is the phylogenetically skewed knowledge of C-to-U RNA editing in chloroplasts. The performance of a lineage is associated with the abundance of data, and especially the number of organisms in that lineage with systematically determined editing sites (Figure 1). The performance for angiosperms is better than that for gymnosperms, as angiosperm data is much more comprehensive for more

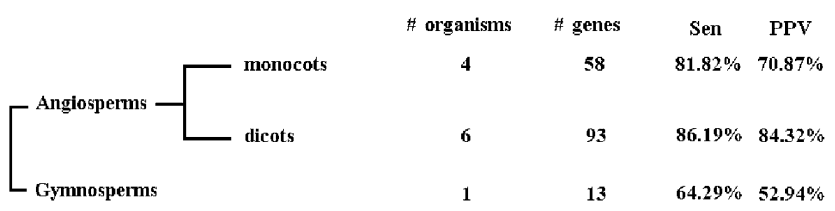

\section{Figure I}

Phylogenetically skewed knowledge of chloroplast Cto-U RNA editing sites. Current knowledge of chloroplast C-to-U RNA editing sites is phylogenetically skewed. The performance of CURE-Chloroplast on different lineages of seed plants is associated with the abundance of data relating to that lineage. The column "\# organisms" refers to the number of organisms in the corresponding lineage. The column "\# genes" refers to the total number of edited genes. organisms. Although the performance for gymnosperms is also very good, it is not as good as that for angiosperms, as the data for gymnosperms is not as abundant as the data for angiosperms. Because these two organisms (Adiantum capillus-veneris and Anthoceros formosae) are the only organism with comprehensive C-to-U RNA editing information in the corresponding lineage, the low performance is expected. When the editing sites of more organisms in these lineages are determined, the performance of CURE-Chloroplast is expected to improve. However, we have to emphasize that CURE-Chloroplast is currently only developed for seed plants.

\section{Conclusion}

CURE-Chloroplast predicts C-to-U RNA editing sites in the chloroplasts of seed plants with quite well performance. The predictive result is expected to improve as more data becomes available. The online service allows the processing of the entire chloroplast genome sequence. Although the predictive ability of CURE-Chloroplast is currently restricted within the seed plant lineage, CUREChloroplast is still a useful tool for studying C-to-U RNA editing in chloroplasts.

\section{Availability and requirements}

Project name: CURE-Chloroplast

Project home page: http://bioinfo.au.tsinghua.edu.cn/ pure

Operating system(s): Online service, platform independent

Programming languages: Java, PHP, JavaScript

Other requirements: The web browser must support JavaScript

License: Free

Restrictions for non-academic use: Please contact the authors before non-academic application 


\section{Authors' contributions}

PD designed the algorithm, carried out the programming, implemented the online service, analyzed the results and partially wrote the manuscript. LJ collected the data, prepared the dataset, carried out the evaluation and optimization of the system, analyzed the results and partially wrote the manuscript. YL directed the entire study, analyzed the results and partially wrote the manuscript.

\section{Additional material}

\section{Additional file 1}

RNAE performance on seed plants. The prediction performance comparison between CURE-Chloroplast and RNAE.

Click here for file

[http://www.biomedcentral.com/content/supplementary/14712105-10-135-S1.pdf]

\section{Additional file 2}

CURE algorithm document. The description of CURE algorithm in details.

Click here for file

[http://www.biomedcentral.com/content/supplementary/14712105-10-135-S2.pdf

\section{Additional file 3}

ROC-like curve on Arabidopsis thaliana. The analysis of CURE-Chloroplast prediction performance under different algorithm parameters. Click here for file

[http://www.biomedcentral.com/content/supplementary/14712105-10-135-S3.pdf]

\section{Acknowledgements}

We thank Dr. John Gray for providing the chloroplast genome of pea. We also thank Dr. Masahiro Sugiura for providing the RNA editing information of pea chloroplast genes. This work was partially supported by the National Nature Science Foundation of China (Grant Nos. 60572086 and 60775002).

\section{References}

I. Mulligan RM, Williams MA, Shanahan MT: RNA editing site recognition in higher plant mitochondria. I Hered 1999 90(3):338-344.

2. Rajasekhar VK, Mulligan RM: RNA Editing in Plant Mitochondria: [alpha]-Phosphate Is Retained during C-to-U Conversion in mRNAs. Plant Cell 1993, 5(1 2): 1843-1852.

3. Yu W, Schuster W: Evidence for a site-specific cytidine deamination reaction involved in C to $U$ RNA editing of plant mitochondria. J Biol Chem I995, 270(31): I8227-I8233.

4. Giege P, Brennicke A: RNA editing in Arabidopsis mitochondria effects 44 I C to U changes in ORFs. Proc Natl Acad Sci USA I999, 96(26): $15324-15329$.

5. Handa $\mathrm{H}$ : The complete nucleotide sequence and RNA editing content of the mitochondrial genome of rapeseed (Brassica napus L.): comparative analysis of the mitochondrial genomes of rapeseed and Arabidopsis thaliana. Nucleic Acids Res 2003, 3 I (20):5907-5916.

6. Notsu Y, Masood S, Nishikawa T, Kubo N, Akiduki G, Nakazono M, Hirai $A$, Kadowaki $K$ : The complete sequence of the rice (Oryza sativa L.) mitochondrial genome: frequent DNA sequence acquisition and loss during the evolution of flowering plants. Mol Genet Genomics 2002, 268(4):434-445.
7. Mower JP, Palmer JD: Patterns of partial RNA editing in mitochondrial genes of Beta vulgaris. Mol Genet Genomics 2006, 276(3):285-293.

8. Maier RM, Neckermann K, Igloi GL, Kossel H: Complete sequence of the maize chloroplast genome: gene content, hotspots of divergence and fine tuning of genetic information by transcript editing. J Mol Biol |995, 25 I (5):6|4-628.

9. Wakasugi T, Hirose T, Horihata M, Tsudzuki T, Kossel H, Sugiura M: Creation of a novel protein-coding region at the RNA level in black pine chloroplasts: the pattern of RNA editing in the gymnosperm chloroplast is different from that in angiosperms. Proc Natl Acad Sci USA 1996, 93(16):8766-8770.

10. Hirose T, Kusumegi T, Tsudzuki T, Sugiura M: RNA editing sites in tobacco chloroplast transcripts: editing as a possible regulator of chloroplast RNA polymerase activity. Mol Gen Genet 1999, 262(3):462-467.

II. Schmitz-Linneweber C, Regel R, Du TG, Hupfer H, Herrmann RG, Maier RM: The plastid chromosome of Atropa belladonna and its comparison with that of Nicotiana tabacum: the role of RNA editing in generating divergence in the process of plant speciation. Mol Biol Evol 2002, 19(9):1602-1612.

12. Tillich M, Funk HT, Schmitz-Linneweber C, Poltnigg P, Sabater B, Martin M, Maier RM: Editing of plastid RNA in Arabidopsis thaliana ecotypes. Plant J 2005, 43(5):708-7I5.

13. Covello PS, Gray MW: RNA editing in plant mitochondria. Nature 1989, 34 I (6243):662-666.

14. Gualberto JM, Lamattina L, Bonnard G, Weil JH, Grienenberger JM: RNA editing in wheat mitochondria results in the conservation of protein sequences. Nature 1989, 34 I (6243):660-662.

15. Hiesel R, Wissinger B, Schuster W, Brennicke A: RNA editing in plant mitochondria. Science 1989, 246(4937): |632-1634.

16. Schuster $W$, Brennicke A: RNA editing makes mistakes in plant mitochondria: editing loses sense in transcripts of a rps 19 pseudogene and in creating stop codons in coxl and rps 3 mRNAs of Oenothera. Nucleic Acids Res 1991, 19(24):6923-6928.

17. Shikanai T: RNA editing in plant organelles: machinery, physiological function and evolution. Cell Mol Life Sci 2006, 63(6):698-708.

18. Heller WP, Hayes ML, Hanson MR: Cross-competition in editing of chloroplast RNA transcripts in vitro implicates sharing of trans-factors between different $\mathbf{C}$ targets. J Biol Chem 2008, 283(I 2):73|4-73|9.

19. Hayes ML, Hanson MR: Identification of a sequence motif critical for editing of a tobacco chloroplast transcript. RNA 2007, I3(2):28|-288.

20. Hayes ML, Reed ML, Hegeman CE, Hanson MR: Sequence elements critical for efficient RNA editing of a tobacco chloroplast transcript in vivo and in vitro. Nucleic Acids Res 2006, 34(I3):3742-3754.

21. Chateigner-Boutin AL, Hanson MR: Developmental co-variation of RNA editing extent of plastid editing sites exhibiting similar cis-elements. Nucleic Acids Res 2003, 3 I ( I 0):2586-2594.

22. Chateigner-Boutin AL, Hanson MR: Cross-competition in transgenic chloroplasts expressing single editing sites reveals shared cis elements. Mol Cell Biol 2002, 22(24):8448-8456.

23. Okuda K, Myouga F, Motohashi R, Shinozaki K, Shikanai T: Conserved domain structure of pentatricopeptide repeat proteins involved in chloroplast RNA editing. Proc Natl Acad Sci USA 2007, 104(19):8|78-8183.

24. Kotera E, Tasaka M, Shikanai T: A pentatricopeptide repeat protein is essential for RNA editing in chloroplasts. Nature 2005, 433(7023):326-330.

25. de Longevialle AF, Meyer EH, Andres C, Taylor NL, Lurin C, Millar $\mathrm{AH}$, Small ID: The pentatricopeptide repeat gene OTP43 is required for trans-splicing of the mitochondrial nad I Intron I in Arabidopsis thaliana. Plant Cell 2007, I 9( I 0):3256-3265.

26. Rudinger M, Polsakiewicz M, Knoop V: Organellar RNA editing and plant-specific extensions of pentatricopeptide repeat proteins in jungermanniid but not in marchantiid liverworts. Mol Biol Evol 2008, 25(7): |405-I4|4.

27. Miyamoto T, Obokata J, Sugiura M: A site-specific factor interacts directly with its cognate RNA editing site in chloroplast transcripts. Proc Natl Acad Sci USA 2004, 10 I (1):48-52.

28. Hirose T, Sugiura M: Involvement of a site-specific trans-acting factor and a common RNA-binding protein in the editing of chloroplast mRNAs: development of a chloroplast in vitro RNA editing system. EMBO J 200I, 20(5): I| |44-| I52. 
29. Cummings MP, Myers DS: Simple statistical models predict Cto-U edited sites in plant mitochondrial RNA. BMC Bioinformatics 2004, 5: 132.

30. Thompson J, Gopal S: Correction: genetic algorithm learning as a robust approach to RNA editing site prediction. BMC Bioinformatics 2006, 7:406.

31. Thompson J, Gopal S: Genetic algorithm learning as a robust approach to RNA editing site prediction. BMC Bioinformatics 2006, 7:145

32. Mower JP: PREP-Mt: predictive RNA editor for plant mitochondrial genes. BMC Bioinformatics 2005, 6:96.

33. Du P, Li Y: Prediction of C-to-U RNA editing sites in plant mitochondria using both biochemical and evolutionary information. J Theor Biol 2008, 253(3):579-586.

34. Yura K, Miyata Y, Arikawa T, Higuchi M, Sugita M: Characteristics and prediction of RNA editing sites in transcripts of the Moss Takakia lepidozioides chloroplast. DNA Res 2008, I 5(5):309-32I.

35. Corneille S, Lutz K, Maliga P: Conservation of RNA editing between rice and maize plastids: are most editing events dispensable? Mol Gen Genet 2000, 264(4):419-424.

36. Calsa Junior T, Carraro DM, Benatti MR, Barbosa AC, Kitajima JP, Carrer $\mathrm{H}$ : Structural features and transcript-editing analysis of sugarcane (Saccharum officinarum L.) chloroplast genome. Curr Genet 2004, 46(6):366-373.

37. Inada M, Sasaki T, Yukawa M, Tsudzuki T, Sugiura M: A systematic search for RNA editing sites in pea chloroplasts: an editing event causes diversification from the evolutionarily conserved amino acid sequence. Plant Cell Physiol 2004, 45(II): $1615-1622$.

38. Zeng WH, Liao SC, Chang CC: Identification of RNA editing sites in chloroplast transcripts of Phalaenopsis aphrodite and comparative analysis with those of other seed plants. Plant Cell Physiol 2007, 48(2):362-368.

39. He T, Du P, Li Y: dbRES: a web-oriented database for annotated RNA editing sites. Nucleic Acids Res 2007:D I 4 I- I 44.

40. Picardi E, Regina TM, Brennicke A, Quagliariello C: REDIdb: the RNA editing database. Nucleic Acids Res 2007:DI73-I77.

4I. Hung J-H, Wang W-C, Huang H-D: Systematic Identification and Repository of RNA Editing Site in Human Genome. International Computer Symposium 2007: I386-139|.

42. Blanchette M, Kent WJ, Riemer C, Elnitski L, Smit AF, Roskin KM, Baertsch R, Rosenbloom K, Clawson H, Green ED, et al.: Aligning multiple genomic sequences with the threaded blockset aligner. Genome Res 2004, 14(4):708-7/5.

43. Funk HT, Berg S, Krupinska K, Maier UG, Krause K: Complete DNA sequences of the plastid genomes of two parasitic flowering plant species, Cuscuta reflexa and Cuscuta gronovii. BMC Plant Biol 2007, 7:45.

44. Wolf PG, Rowe CA, Hasebe M: High levels of RNA editing in a vascular plant chloroplast genome: analysis of transcripts from the fern Adiantum capillus-veneris. Gene 2004, 339:89-97.

45. Kugita M, Yamamoto Y, Fujikawa T, Matsumoto T, Yoshinaga K: RNA editing in hornwort chloroplasts makes more than half the genes functional. Nucleic Acids Res 2003, 3 I (9):24I7-2423.

46. Tillich M, Lehwark P, Morton BR, Maier UG: The evolution of chloroplast RNA editing. Mol Biol Evol 2006, 23(10):1912-1921.
Publish with Bio Med Central and every scientist can read your work free of charge

"BioMed Central will be the most significant development for disseminating the results of biomedical research in our lifetime. "

Sir Paul Nurse, Cancer Research UK

Your research papers will be:

- available free of charge to the entire biomedical community

- peer reviewed and published immediately upon acceptance

- cited in PubMed and archived on PubMed Central

- yours - you keep the copyright

Submit your manuscript here:

http://www.biomedcentral.com/info/publishing_adv.asp
BioMedcentral 